\title{
Summed rest score in gated myocardial perfusion imaging is a good predicator for treatment-related cardiotoxicity after anthracycline chemotherapy in patients with diffuse large B-cell lymphoma
}

\author{
YAN LIN $^{1 *}$, JIANFENG WANG ${ }^{2 *}$, MIN XU $^{3 *}$, CHUN QIU $^{2}$, PENG XU $^{1}$, LIMEI SHANG $^{1}$, \\ BAI HE ${ }^{1}$, FEI WANG ${ }^{1}$, YANHUA YUE ${ }^{1}$, YANTING GUO ${ }^{1},{\text { FENG } \text { LI }^{1} \text {, WEIMIN DONG }}^{1}$, \\ XIAOBAO XIE ${ }^{1}$, YUETAO WANG ${ }^{2}$ and WEIYING GU ${ }^{1}$ \\ Departments of ${ }^{1}$ Hematology, ${ }^{2}$ Nuclear Medicine and ${ }^{3}$ Echocardiography, The Third Affiliated \\ Hospital of Soochow University, Changzhou, Jiangsu 213003, P.R. China
}

Received March 13, 2020; Accepted September 11, 2020

DOI: $10.3892 / \mathrm{ol} .2020 .12194$

\begin{abstract}
Anthracycline chemotherapy is commonly used in the treatment of diffuse large B-cell lymphoma (DLBCL). Treatment-related cardiotoxicity (TRC) is defined as when the patient is identified to have one of the following clinical manifestations: Symptomatic heart failure, cardiac death, arrhythmia, infarction, a decrease in left ventricular ejection fraction (LVEF) of $>15 \%$ from baseline or a decrease in LVEF of $>10$ to $<50 \%$. TRC may induce severe cardiac failure or cardiac arrhythmia as the main cause of death. The present study aimed to investigate the prognostic value of the summed rest score (SRS) in gated myocardial perfusion imaging (G-MPI) for the early detection of TRC caused by anthracycline chemotherapy in patients with DLBCL. A total of 36 DLBCL patients were enrolled in the present study, and a series of parameters were compared at baseline and after chemotherapy. According to the occurrence of TRC during the observation period, the patients were divided into two groups, and parameters associated with cardiac function were compared. The SRS in G-MPI and the corrected QT interval in the electrocardiogram were significantly different before
\end{abstract}

Correspondence to: Professor Weiying Gu, Department of Hematology, The Third Affiliated Hospital of Soochow University, 185 Juqian, Changzhou, Jiangsu 213003, P.R. China

E-mail: guweiying2017@126.com

Professor Yuetao Wang, Department of Nuclear Medicine, The Third Affiliated Hospital of Soochow University, 185 Juqian, Changzhou, Jiangsu 213003, P.R. China

E-mail: yuetao-w@163.com

${ }^{*}$ Contributed equally

Key words: diffuse large B-cell lymphoma, summed rest score, gated myocardial perfusion imaging, treatment-related cardiotoxicity and after chemotherapy ( $\mathrm{P}=0.012$ and $\mathrm{P}=0.015$, respectively). By comparing parameters associated with cardiac function between the TRC group $(n=22)$ and the no-TRC group $(\mathrm{n}=14)$, it was found that only SRS was significantly different $(\mathrm{P}=0.012)$. Multivariate logistic regression analysis showed that the SRS level was the only independent predicator for TRC ( $\mathrm{P}=0.018$; HR, 6.053; 95\% CI, 1.364-26.869). Receiver operating characteristic curve analysis identified an optimal SRS cutoff of $>1$ for predicting TRC after anthracycline chemotherapy $(\mathrm{P}<0.001)$. Overall, the G-MPI SRS level was an early indicator for TRC surveillance in patients with DLBCL after anthracycline chemotherapy. The application of G-MPI SRS in clinical practice may contribute to early treatment and a subsequent decrease in mortality caused by such cardiovascular complications.

\section{Introduction}

Diffuse large B-cell lymphoma (DLBCL) is the most common type of non-Hodgkin's lymphoma worldwide, representing $30-40 \%$ of all cases among different geographic regions (1). Although the use of anti-CD20 monoclonal antibodies has significantly improved the survival of patients with DLBCL, traditional chemotherapy is still indispensable for the treatment of this lymphoma. Due to their high efficacy and broad spectrum of activity, anthracyclines, such as Adriamycin and epirubicin, play an important role in first-line chemotherapeutic regimens for DLBCL (2).

Anthracyclines are commonly associated with cardiotoxicity, occurring with an incidence of $\sim 9 \%(3,4)$. The prognosis of anthracycline-induced heart failure remains poor, with a 2-year mortality rate of $60 \%$ (5). Clinical guidelines suggest limiting the maximum cumulative dose to reduce the incidence of cardiotoxicity while maintaining the maximum antitumor effect (6).

Anthracycline-induced cardiotoxicity most likely occurs at the time of exposure, while the symptoms of cardiotoxicity associated with heart failure may be masked for several years. 
Usually, anticongestive treatment is only given once clinical manifestations show that the compensatory mechanism of the heart is no longer adequate, leading to a seriously deteriorated prognosis (7). With early recognition and treatment initiation, the prognosis of patients can be significantly improved (8). Acute cardiotoxic side effects are rare and usually reversible. Late-onset cardiomyopathy, which is difficult to treat and associated with a poor prognosis, is more frequent. Therefore, it is highly necessary to identify an appropriate and sensitive approach for the early detection of myocardial injury to improve the quality of life and prolong the survival of patients with DLBCL.

The left ventricular ejection fraction (LVEF) refers to the ratio of stroke volume divided by the left ventricular end diastolic (LVED) volume (9). Notably, LVEF remains used as a classic index to evaluate systolic function, albeit being highly dependent on preload and afterload conditions, and it is a late marker of cardiotoxicity (10). The activation of the sympathetic nervous system in patients with heart failure maintains the normal LVEF (7). It may be a late finding to detect a decrease in LVEF after cancer treatment. Therefore, early markers associated with myocardial dysfunction are highly desired (11-13).

The risk of cardiotoxicity may be reduced if the administration of an angiotensin-converting enzyme inhibitor or angiotensin receptor blocker, $\beta$-blocker and/or statin starts early (11). In order to reduce the risk of cardiotoxicity and enhance the cardiac protection strategies, researchers have made good efforts to find specific and sensitive markers of early LV dysfunction, including cardiac biomarkers and advanced imaging modalities (14,15).

Compared with other traditional methods such as echocardiography, cardiac CT and cardiac MRI, gated myocardial perfusion imaging (G-MPI) is a novel and sensitive method that can identify the small myocardial injury caused by cancer therapeutics $(16,17)$. With regard to G-MPI, the majority of studies focused on patients who underwent radiation therapy, and a higher percentage of perfusion defects with associated wall motion abnormalities after radiation therapy was found $(18,19)$. The summed rest score (SRS), summed motion score (SMS) and summed thickening score (STS) are quantitative parameters obtained by quantitative analysis of resting G-MPI. The SRS has been used to evaluate the degree of damage to myocardial perfusion at rest, and has higher diagnostic sensitivity and accuracy compared with visual evaluation (20). Anthracycline chemotherapeutics can damage myocardial cells, meaning that the resultant myocardial perfusion can also be abnormal. However, whether quantitative analysis of resting G-MPI compared with LVEF can detect the chemotherapy-induced myocardial injury in lymphoma patients at an earlier stage remains largely unexplored.

The present study aimed to investigate the prognostic value of G-MPI parameters in the early detection of impaired myocardial function in patients with DLBCL receiving anthracycline chemotherapy.

\section{Materials and methods}

Study design. A total of 36 patients, who were newly diagnosed with DLBCL in the Department of Hematology of the Third Affiliated Hospital of Soochow University (Changzhou, China), were enrolled in the present analysis between March 2016 and July 2019. Patients with any heart disease at baseline examination were excluded. The exclusion criteria included the following: Abnormalities on electrocardiogram (ECG) or echocardiography, plus any abnormalities in myocardial markers or brain natriuretic peptide (BNP). Patients who met any of the four criteria were excluded from the study. All DLBCL diagnoses were made based on the histological pathology analysis (21). Histopathological criteria for DLBCL are defined in the World Health Organization 2008 classification of lymphoid neoplasms (22). Clinical staging data were determined according to the Ann Arbor staging classification (23). This study was designed to assess the cardiac function of each patient by serum test, echocardiography and G-MPI, at baseline and after the last cycle of chemotherapy (mean time, 6 months).

All patients were administered with six cycles of an R-CHOP regimen $\left(375 \mathrm{mg} / \mathrm{m}^{2}\right.$ rituximab, $750 \mathrm{mg} / \mathrm{m}^{2}$ cyclophosphamide, $1.4 \mathrm{mg} / \mathrm{m}^{2}$ vincristine, $70 \mathrm{mg} / \mathrm{m}^{2}$ epirubicin or $25 \mathrm{mg} / \mathrm{m}^{2}$ pegylated liposomal doxorubicin, and $100 \mathrm{mg}$ prednisone for 5 days). The R-CHOP regimen was administered every 3 or 4 weeks according to the hematopoietic recovery of the patient. A series of serum parameters were detected before and after chemotherapy, including fast blood sugar (FBS; mmol/l), total cholesterol (TC; mmol/l), high-density lipoprotein (mmol/l), low-density lipoprotein (LDL; mmol/l), triacylglycerol (mmol/l), alanine aminotransferase (U/1), creatinine (mmol/l), D-Dimer (mg/l), white blood cells (x10 $9 / 1)$, hemoglobin $(\mathrm{g} / \mathrm{l})$, platelets $\left(\mathrm{x} 10^{9} / \mathrm{l}\right)$, red blood cell distribution width (\%), $\beta 2$-microglobulin (mg/l), C-reactive protein $(\mathrm{mg} / \mathrm{l})$, procalcitonin $(\mathrm{ng} / \mathrm{ml}), \mathrm{BNP}(\mathrm{pg} / \mathrm{ml})$, aspartate transaminase (U/1), lactic dehydrogenase (U/l), creatine kinase (CK; U/l), CK-MB (ng/ml), troponin ( $\mu \mathrm{g} / \mathrm{l})$ and myoglobin $(\mathrm{ng} / \mathrm{ml})$.

All procedures were in accordance with the ethical standards of the responsible human experimentation committee (institutional and national) and the Declaration of Helsinki (1975), as amended in 2008. Patient samples and the clinical data were obtained with approval from the Ethical Committee of The Third Affiliated Hospital of Soochow University (approval number, CL022-01), and written informed consent was obtained according to the institutional guidelines.

Evaluation of cardiac function. The cardiac function of each patient was evaluated using a series of serum indicators, ECG, echocardiography and G-MPI. Treatment-related cardiotoxicity (TRC) was defined as when the patient was identified to have one of the following clinical manifestations: Symptomatic heart failure, cardiac death, arrhythmia, infarction, a decrease in left ventricular ejection fraction (LVEF) of $>15 \%$ from baseline or a decrease in LVEF of $>10$ to $<50 \%$. All other cases were defined as no-TRC $(24,25)$.

The serum indicators for cardiac function included brain natriuretic peptide, troponin, aspartate transaminase, lactic dehydrogenase, creatine kinase (CK), CK-MB isoenzyme and myoglobin. The levels of all these indicators were determined using an XE-2100 automated analyzer (Sysmex Co., Ltd.).

Heart rate, PR interval, QRS interval, QT interval and QTc interval measurements were determined by a 12-lead ECG ((FX-8322; Fukuda Sangyo, Co., Ltd.). Echocardiographic 
examinations were performed using the ultrasonic diagnostic apparatus (Vivid E9; GE Healthcare) with a 3.5-MHz transducer. Reports were made according to the American Society of Echocardiography guidelines $(26,27)$. The indicators relevant to research included left atrial diameter, left ventricular posterior wall thickness, left ventricular end systolic diameter, interventricular septal thickness, LVED diameter and LVEF.

Resting G-MPI was performed using a single-photon emission CT/CT scanner (Symbia T16; Siemens Healthineers), which was equipped with a low-energy high-resolution collimator. Briefly, the patient was intravenously injected with $740-925 \mathrm{MBq}$ for resting studies, and the radiochemical purity was $>95 \%$. At $60 \mathrm{~min}$ after the injection, the electrodes were placed upon the skin above the heart of the patient with two detectors set at $90^{\circ}$. Images were acquired every $35 \mathrm{sec}$ and every $6^{\circ}$ clockwise from $45^{\circ}$ at the right anterior oblique position to $45^{\circ}$ at the left anterior oblique position, with an acquisition matrix of $128 \times 128$ and a magnification of 1.45. Subsequently, the gated acquisition was performed with electrocardiographic R-wave as the acquisition trigger signal and eight frames/R-R interval. The planar images were reconstructed by a Flash $3 \mathrm{D}^{\mathrm{TM}}$ iterative method (28) (16 iterations and two subsets) and reoriented to obtain LV short-axis, horizontal long-axis and vertical long-axis images. The left venticle was divided into 17 segments using QPS and QGS quantitative analysis software (QPS \& QGS 2009; Cedars-Sinai Medical Center). The QPS was used to analyze myocardial perfusion. The segmental radiotracer activity in the resting scans was scored according to a standard 5-point scale $(0$, normal; 1 , mild; 2 , moderate; 3 , severe; and 4 , absent activity) and summed to generate an SRS (29). QGS was used to obtain the LVEF, SMS and STS.

Statistical analysis. All statistical analyses in this study were performed using SPSS version 24.0 statistical software (IBM Corp.) and MedCalc software version 15.11.4 (MedCalc Software bvba). Normally distributed data are expressed as the mean \pm standard error, while data without a normal distribution are presented as the median (interquartile range). Paired t-tests, Wilcoxon's matched pairs test, independent samples t-tests and Mann-Whitney U tests were used to compare the differences between groups depending on the type of data. Proportional differences were evaluated with the $\chi^{2}$ test or Fisher's exact test in the supplementary tables. The optimal cutoff value for the association of the SRS level with TRC after anthracycline chemotherapy was determined based on the result of receiver operating characteristic (ROC) curve analysis. The initial univariate logistic regression analysis was followed by a multivariate logistic regression model using stepwise selection to identify univariate predictors of TRC. $\mathrm{P}<0.05$ was considered to indicate a statistically significant difference.

\section{Results}

Baseline patient characteristics. Between March 2016 and July 2019, 36 patients with DLBCL were enrolled in the present study. Table I lists the baseline clinical characteristics of these patients. The mean age of the cohort was 58 years (range, 27-72 years), and there were 20 males and 16 females. The body mass index (BMI) of these patients was 22.854 \pm 0.636 .
Table I. Baseline of the clinical characteristics of the 36 patients with diffuse large B-cell lymphoma enrolled in the present study.

\begin{tabular}{lc}
\hline Characteristics & Value \\
\hline Median age (range), years & $58(27-82)$ \\
Sex, n (\%) & \\
Male & $20(56)$ \\
Female & $16(44)$ \\
BMI $^{\mathrm{a}}$ & $22.854 \pm 0.636$ \\
Comorbidity, n (\%) & \\
Heart disease & $0(0)$ \\
Hypertension & $8(22)$ \\
Diabetes & $5(14)$ \\
Hypercholesteremia & $10(28)$ \\
Hypertriglyceridemia & $10(28)$ \\
Clinical stage, n (\%) & \\
I-II & $10(28)$ \\
III-IV & $26(72)$ \\
B-symptoms, n (\%) & $10(28)$
\end{tabular}

${ }^{\mathrm{a}}$ Mean \pm standard deviation. BMI, body mass index.

At baseline, all of the recruited patients had no comorbidity of heart disease, while 8 patients had hypertension, 5 patients had diabetes, and 10 patients suffered from hypercholesteremia and hypertriglyceridemia. Moreover, 10 patients $(28 \%)$ were found to be in the early stage of lymphoma according to Ann Arbor classification (I-II), and 26 patients (72\%) were found to be in the late stage of lymphoma (III-IV). In addition, 10 patients $(28 \%)$ were diagnosed with B symptoms.

Comparison of serum, echocardiography, ECG and G-MPI parameters at baseline and after chemotherapy. As listed in Table II, by comparing the serum parameters of these patients before and after six courses of R-CHOP chemotherapy, it was found that the levels of FBS $(\mathrm{P}=0.002)$, TC $(\mathrm{P}=0.03)$ and $\mathrm{LDL}$ $(\mathrm{P}=0.001)$ were significantly increased, and that the hemoglobin level was significantly higher $(\mathrm{P}=0.034)$ after chemotherapy.

Considering the cumulative cardiotoxicity of anthracyclines, cardiac function-related serum indicators of these patients were compared at the time of initial treatment and after chemotherapy. There were no significant differences, as listed in Table II ( $\mathrm{P}>0.05)$.

As listed in Table III, echocardiographic and ECG parameters of these patients were compared at baseline and after chemotherapy. No significant differences in these parameters were found by echocardiography, including LVEF ( $\mathrm{P}>0.05)$. Notably, it was found that the QTc interval $(\mathrm{P}=0.015)$ was significantly increased after chemotherapy. By using the novel G-MPI method, it was found that SRS, the indicator representing myocardial perfusion, was significantly higher after chemotherapy $(\mathrm{P}=0.012)$ (Table IV; Fig. 1).

Association between SRS and TRC. TRC was defined using similar criteria as previously described $(20,23)$, including 
Table II. Comparison of plasma markers before and after chemotherapy.

\begin{tabular}{|c|c|c|c|}
\hline Plasma markers & Before chemotherapy & After chemotherapy & P-value \\
\hline $\mathrm{FBS}, \mathrm{mmol} / \mathrm{l}$ & $4.980(0.870)$ & $5.300(1.320)$ & 0.002 \\
\hline $\mathrm{TC}, \mathrm{mmol} / \mathrm{l}$ & $4.287 \pm 0.156$ & $4.716 \pm 0.159$ & 0.030 \\
\hline $\mathrm{HDL}, \mathrm{mmol} / \mathrm{l}$ & $1.076 \pm 0.057$ & $1.166 \pm 0.053$ & 0.073 \\
\hline LDL, mmol/1 & $2.521 \pm 0.108$ & $2.847 \pm 0.134$ & 0.001 \\
\hline $\mathrm{TG}, \mathrm{mmol} / \mathrm{l}$ & $1.036(0.600)$ & $1.475(0.985)$ & 0.155 \\
\hline ALT, U/1 & $18(19.500)$ & $18(15)$ & 0.665 \\
\hline Creatinine, $\mathrm{mmol} / \mathrm{l}$ & $65.667 \pm 1.709$ & $68.273 \pm 2.029$ & 0.164 \\
\hline D-Dimer, mg/l & $0.505(0.740)$ & $0.395(0.398)$ & 0.275 \\
\hline WBC (x109/1) & $5.060(2.318)$ & $4.51(2.495)$ & 0.139 \\
\hline Hemoglobin, g/l & $121.500(24.250)$ & $125.500(25.500)$ & 0.034 \\
\hline Platelets $\left(\times 10^{9} / 1\right)$ & $194.794 \pm 11.824$ & $187.147 \pm 10.871$ & 0.464 \\
\hline RDW, \% & $13.100(1.775)$ & $13.500(1.875)$ & 0.174 \\
\hline$\beta 2-\mathrm{MG}, \mathrm{mg} / \mathrm{l}$ & $1.798 \pm 0.125$ & $1.854 \pm 0.091$ & 0.706 \\
\hline $\mathrm{CRP}, \mathrm{mg} / \mathrm{l}$ & $7.250(42.250)$ & $4.950(5.300)$ & 0.177 \\
\hline $\mathrm{PCT}, \mathrm{ng} / \mathrm{ml}$ & $0.053(0.071)$ & $0.069(0.085)$ & 0.655 \\
\hline $\mathrm{BNP}, \mathrm{pg} / \mathrm{ml}$ & $67.500(64.250)$ & $36.000(57.388)$ & 0.060 \\
\hline AST, U/1 & $24(11)$ & $26(11)$ & 0.820 \\
\hline LDH, U/1 & $587.136 \pm 51.855$ & $557.136 \pm 28.157$ & 0.527 \\
\hline $\mathrm{CK}, \mathrm{U} / \mathrm{l}$ & $49(24.500)$ & $55(38)$ & 0.509 \\
\hline $\mathrm{CK}-\mathrm{MB}, \mathrm{ng} / \mathrm{ml}$ & $0.700(0.700)$ & $0.9(0.800)$ & 0.149 \\
\hline Troponin, $\mu \mathrm{g} / \mathrm{l}$ & $0.001(0.003)$ & $0.003(0.002)$ & 0.142 \\
\hline Myoglobin, ng/ml & $15.100(8.800)$ & $15.800(10.050)$ & 0.765 \\
\hline
\end{tabular}

Table III. Comparison of markers in echocardiogram and electrocardiogram before and after chemotherapy.

\begin{tabular}{lccc}
\hline Marker & Before chemotherapy & After chemotherapy & P-value \\
\hline Echocardiogram & & & \\
LAD, mm & $35.389 \pm 1.462$ & $34.500 \pm 0.883$ & 0.371 \\
LVEDD, mm & $46.000 \pm 0.957$ & $45.667 \pm 0.886$ & 0.674 \\
LVESD, mm & $31(5)$ & $30.500(2.500)$ & 0.754 \\
IVST, mm & $9(1)$ & $9(1)$ & 0.604 \\
LVPWT, mm & $9(0.500)$ & $62.500 \pm 0.445$ & 0.964 \\
LVEF, \% & $62.889 \pm 0.593$ & & 0.369 \\
Electrocardiogram & $73(18)$ & $179(10.500)$ & 0.102 \\
Heart rate, bpm & $153.500(28)$ & $100(13.000)$ & 0.475 \\
PR interval, msc & $100(10.500)$ & $372.636 \pm 4.482$ & 0.283 \\
QRS interval, msec & $375.970 \pm 5.607$ & $429.344 \pm 3.349$ & 0.549 \\
QT interval, msec & $421.250 \pm 3.775$ & 0.015 \\
QTc interval, msec & & & \\
\hline
\end{tabular}

Data are presented as mean \pm standard error or median (interquartile range). Paired t-tests were used in the analyses of LAD, LVED, DLVEF, QT interval and QTc interval. Wilcoxon matched pairs tests were used in the analyses of LVESD, IVST, LVPWT, heart rate, PR interval and QRS interval. LAD, left atrial diameter; LVEDD, left ventricular end diastolic diameter; LVSED, left ventricular end systolic diameter; IVST, interventricular septal thickness; LVPWT, left ventricular posterior wall thickness; LVEF, left ventricular ejection fraction; QTc, corrected QT.

a decrease in LVEF of $>15 \%$ from baseline, a decrease in LVEF of $>10$ to $<50 \%$, symptomatic heart failure, arrhythmia, infarction or cardiac death. According to these criteria and the characteristics of patients during observation, all the patients 
Table IV. Comparison of markers associated with myocardial perfusion in gated myocardial perfusion imaging before and after chemotherapy.

\begin{tabular}{lccr}
\hline Markers & Before chemotherapy & After chemotherapy & P-value \\
\hline SRS & 2 & 3 & 0.012 \\
SMS rest & 0 & 0 & 0.900 \\
STS rest & 0 & 0 & 0.858 \\
EF rest & $65.514 \pm 1.608$ & $64.714 \pm 1.746$ & 0.582 \\
\hline
\end{tabular}

Data are presented as mean \pm standard error. Paired t-test was used in the analysis of EF rest. Wilcoxon matched pairs tests were used in the analyses of SRS, SMS rest and STS rest. SRS, summed rest score; SMS, summed motion score; STS, Summed thickening score.

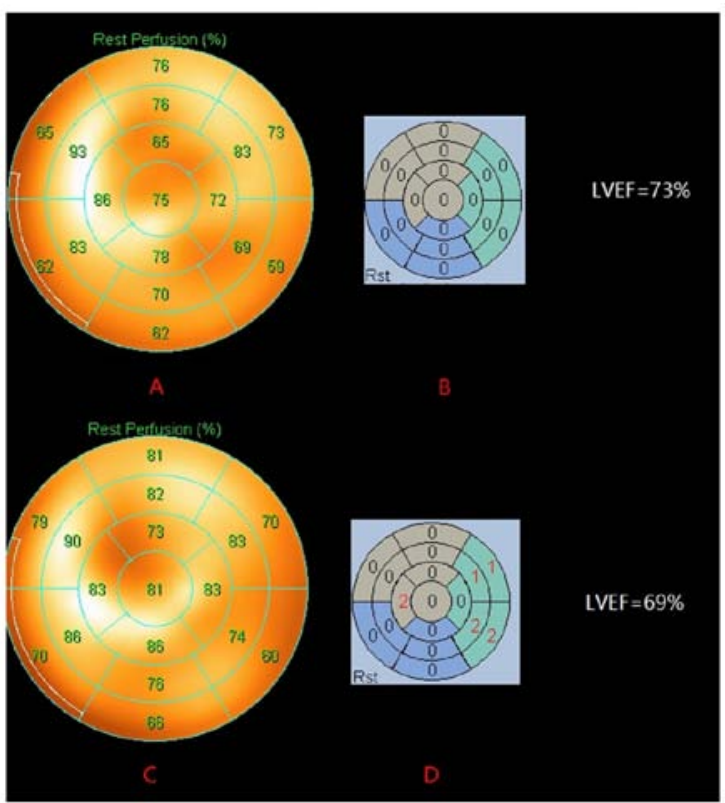

Figure 1. A representative case showing a significant change in G-MPI SRS in a DLBCL patient before and after R-CHOP chemotherapy. The patient was a 69-year-old male. The LV systolic function was normal before chemotherapy (LVEF, 73\%), (A) the MPI resting myocardial perfusion bull's eye chart showed an even distribution of imaging agent in each wall of the LV, and (B) SRS was 0 , indicating that the $\mathrm{LV}$ myocardial perfusion before chemotherapy was good. The LV systolic function was not significantly damaged after six courses of R-CHOP chemotherapy regimens (LVEF, 69\%), (C) while the MPI resting myocardial perfusion bull's eye chart showed uneven distribution compared with that before chemotherapy, and (D) the SRS was 8, revealing that despite no obvious changes in LVEF after anthracycline chemotherapy in patients with DLBCL, LV myocardial perfusion was already abnormal. LV, left ventricular; G-MPI, gated myocardial perfusion imaging; SRS, summed rest score; DLBCL, diffuse large B-cell lymphoma; LVEF, LV ejection fraction.

were divided into the TRC group $(\mathrm{n}=22)$ and the no-TRC group $(\mathrm{n}=14)$. In the TRC group, 1 patient had a myocardial infarction, 1 patient suffered cardiac death, 20 patients had other types of arrhythmia and 4 patients had a decrease in LVEF of $>15 \%$ from baseline or a decrease in LVEF of $>10$ to $<50 \%$. No patients had symptomatic heart failure.

The baseline characteristics of patients in these two groups were compared. Moreover, the plasma markers, echocardiography, ECG and G-MPI parameters of patients after six courses of chemotherapy were also assessed between these two groups (Tables SI-III and V). There was no significant difference in any of aforementioned parameters after chemotherapy,

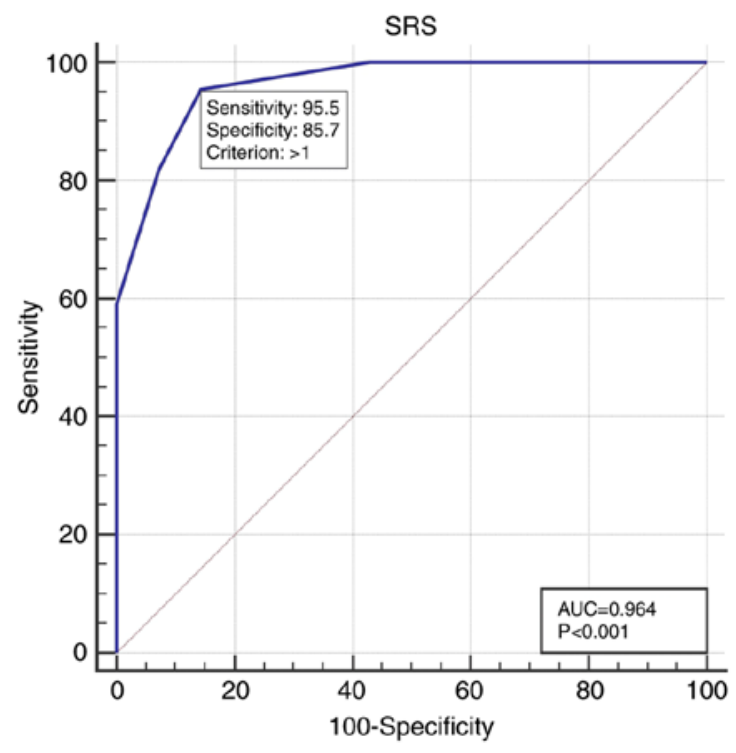

Figure 2. ROC curve analysis to identify the optimal SRS cutoff for predicting TRC. ROC curve analysis identified an optimal SRS cutoff of $>1$ for predicting TRC after anthracycline chemotherapy, with a sensitivity of $95.5 \%$, a specificity of $85.7 \%$ and an AUC of $0.964(\mathrm{P}<0.001)$. ROC, receiver operating characteristic; SRS, summed rest score; TRC, treatment-related cardiotoxicity; AUC, area under the curve.

with the exception of SRS from G-MPI. The median SRS level of the TRC and no-TRC groups was 4 and 0 , respectively $(\mathrm{P}<0.0001)$.

After adjustment for LVEF in echocardiography and QTc interval in ECG, multivariate logistic regression analysis showed that the SRS level was the only independent predictor for TRC (OR, 6.503); 95\% CI, 1.364-26.869; P=0.018; Table VI).

Using ROC curve analysis, an optimal SRS cutoff of $>1$ was identified for predicting TRC, with a sensitivity of $95.5 \%$, a specificity of $85.7 \%$ and an area under the curve (AUC) of 0.964 ( $\mathrm{P}<0.001$; Fig. 2).

\section{Discussion}

To the best of our knowledge, the present study is the first to use the resting G-MPI to evaluate the abnormal changes in LV myocardial perfusion in DLBCL patients before and after chemotherapy. The results showed that the SRS value detected after chemotherapy was significantly higher than that before chemotherapy, indicating that there was abnormal LV perfusion 
Table V. Comparison of markers in gated myocardial perfusion imaging in the no-TRC and TRC groups after chemotherapy.

\begin{tabular}{lccc}
\hline Markers & No TRC & TRC & P-value \\
\hline SRS & $0(1)$ & $4(3.25)$ & $<0.0001$ \\
SMS rest & $0(0.25)$ & $0(1.5)$ & 0.375 \\
STS rest & $0(0)$ & $0(0.5)$ & 0.455 \\
EF rest & $61.357 \pm 2.122$ & $66.952 \pm 2.463$ & 0.118
\end{tabular}

Data are presented as mean \pm standard error. Independent samples t-test was used in the analysis of EF rest. Mann-Whitney tests were used in the analyses of SRS, SMS rest and STS rest. TRC, treatment-related cardiotoxicity; SRS, summed rest score; SMS, summed motion score; STS, Summed thickening score; EF, ejection fraction.

Table VI. Univariate and multivariate logistic regression analyses.

\begin{tabular}{lcccr}
\hline & & \multicolumn{2}{c}{ Multivariate analysis } \\
\cline { 4 - 5 } Variables & Univariate analysis P-value & OR & 95\% CI & P-value \\
\hline Clinical characteristics & & & & $0.709-24.738$ \\
SRS & $<0.0001$ & 6.503 & $0.888-1.086$ & 0.721 \\
QTc & 0.953 & 0.982 & $0.894-1.189$ & 0.673 \\
EF rest & 0.118 & 1.031 & \\
\hline
\end{tabular}

Initial univariate logistic regression analysis was followed by a multivariate logistic regression model using stepwise selection to identify univariate predictors of treatment-related cardiotoxicity. SRS, summed rest score; QTc, correction QT interval; EF, ejection fraction.

after chemotherapy. Logistic multivariate analysis showed that SRS was an independent risk factor for TRC after chemotherapy in patients with DLBCL. ROC curve analysis showed that when SRS was $>1$, the possibility of TRC was high.

The prognosis of patients with DLBCL has been greatly improved due to earlier detection and new, targeted therapeutic drugs. In terms of longer survivorship, a large number of patients with DLBCL have to face the risk of cardiovascular morbidity and mortality, which are caused by the long-term toxicity of anthracycline chemotherap (30-33). Cardiotoxicity caused by anthracycline is often progressive and irreversible, which severely affects the prognosis of patients with DLBCL (14,34,35). Serial echocardiographic evaluation of resting LVEF is still a classic index to monitor the anthracycline-induced cardiac side effects from chemotherapy. However, LVEF tests are limited by their low sensitivity. A normal LVEF level does not mean that there is no cardiotoxicity. The irreversible cardiomyopathy often happens before the observation of a decreased LVEF $(4,7)$. It has been reported that at the early stage of cardiac function impairment, most patients present with subclinical myocardial damage (36). The early detection and treatment of cardiotoxicity, even when asymptomatic, seem to be critical for cardiac function recovery and for reduction in associated adverse cardiac events. There is no significant change in LV function, ECG results or myocardial markers in the early stage of myocardial damage. Only when the myocardial function is severely damaged or the overall heart function is impaired, do abnormal changes become detectable in indicators, such as LV systolic function. In the present study, there was no significant difference in LVEF values before and after chemotherapy in the patients with DLBCL. According to the TRC criteria of the LVEF value, only 4 patients with DLBCL developed LVEF-related TRC after chemotherapy. Univariate and multivariate analyses of TRC showed that LVEF was not an independent risk factor for TRC. The results further confirmed that LVEF could not evaluate the cardiotoxicity caused by anthracycline drugs in the early stage.

The QTc interval on the ECG refers to the QT interval corrected according to heart rate or R-R interval (37). Antineoplastic drugs open sodium and calcium channels by blocking potassium channels, resulting in cardiac repolarization abnormality, and a prolonged QTc interval would be demonstrated on the ECG (38). Drug-induced prolongation of QTc interval has been considered to be a critical risk factor for cardiac toxicity, and it likely leads to palpitations, syncope and sudden cardiac death (SCD) caused by severe ventricular arrhythmias $(35,38,39)$. In a follow-up study consisting of 147 adult survivors who previously suffered from childhood tumors and received anthracycline chemotherapy, Markman et al (14) found that the prolonged QTc interval is associated with the subsequent development of LV dysfunction, suggesting that the QTc interval is an earlier indicator for evaluating the impairment of cardiac function compared with LVEF. The normal range of the QTc interval is $350-450 \mathrm{msec}$ in adult males and 360-460 msec in adult femal (38). Puppe et al (38) highlighted the tripled risk of SCD when the QT interval is $>470$ msec. The study by Porta-Sanchez (36) indicated that the patients should be carefully evaluated to determine whether they should discontinue the usage of all offending drugs when a prolonged QTc interval is detected $(>500 \mathrm{msec}$ or an increase of $>60$ msec greater than baseline) (36). When the 
QTc interval of a patient fluctuates within the normal range, clinicians are prone to lacking the same vigilance in checking for myocardial injury. In addition, considerable variation may be caused by differences in the technical circumstances and non-pathological biological variability. All the aforementioned factors have contributed to the uncertainty of using the ECG QTc interval as an indicator of cardiac toxicity monitoring. In the present study, the mean QTc interval of the patients after anthracycline chemotherapy was $429.344 \mathrm{msec}$. Although there was a significant difference before and after treatment, the mean QTc interval after anthracycline chemotherapy did not reach the upper limit of the normal QTc interval of $450 / 460 \mathrm{msec}$. Moreover, the results of this study showed that the QTc interval was not an independent risk factor for TRC. Therefore, there were still limitations of using the QTc interval as an early indicator of cardiac toxicity.

The mechanisms of anthracycline-induced cardiomyopathies include endoplasmic reticulum stress, calcium dysregulation, activation of the immune system and impairment of progenitor cells, among others $(40,41)$. To date, the most widely cited and accepted mechanism is the formation of reactive oxygen species (ROS), which leads to oxidative stress (42-45). Koleini and Kardami (46) indicated that cardiomyocytes need a large number of healthy functioning mitochondria to product sufficient ATP to maintain contractile function and cell survival. Autophagic cell death and mitophagy have been proposed to be associated with the cardiotoxicity of anthracycline (46). Ichikawa et al (47) found that anthracycline-dependent cardiotoxicity occurs through ROS production and possibly mitochondrial iron accumulation. Mitochondria play a pivotal role in the metabolism and apoptosis of cardiomyocytes. Technetium $99 \mathrm{~m}$ sestamibi (99mTc-MIBI) is a lipophilic cation imaging tracer mainly localized in the mitochondria; it is mainly sequestered within the mitochondria by a huge negative transmembrane potential (48). The distribution of ${ }^{99 \mathrm{~m}} \mathrm{Tc}-\mathrm{MIBI}$ in the myocardium is directly proportional to myocardial blood flow. When the mitochondrial function of cardiomyocytes is impaired, collapse of the mitochondrial membrane potential will lead to quick release of extracted ${ }^{99 \mathrm{~m}}$ Tc-MIBI. Therefore, G-MPI can show abnormal local myocardial perfusion, which can be used for early non-invasive evaluation of abnormal changes in the mitochondrial function of cardiomyocytes (48-50).

SRS is an indicator of G-MPI for the quantitative evaluation of resting myocardial perfusion abnormalities and can directly reflect changes in cardiomyocyte function $(51,52)$. To the best of our knowledge, no studies have reported the quantitative analysis of G-MPI in the early evaluation of myocardial damage caused by chemotherapy. In the present study, ${ }^{99 \mathrm{~m}} \mathrm{Tc}-\mathrm{MIBI}$ G-MPI was used to quantitatively analyze the changes in myocardial perfusion before and after chemotherapy. The results showed that the resting perfusion SRS score in DLBCL patients after chemotherapy was significantly higher than that before chemotherapy. In addition, logistic multivariate analysis we applied and SRS was found to be an independent risk factor for predicting TRC in patients with DLBCL after chemotherapy. ROC curve analysis indicated that an SRS $>1$ had the highest efficiency in predicting TRC (AUC, 0.964; sensitivity, 95.5\%; specificity, $85.7 \%$ ). In other words, when the SRS was $>1$, prompt intervention was required to prevent TRC. In the present study, after six courses of anthracycline-containing chemotherapy, all of the DLBCL patients with TRC developed arrhythmia (22/22), and only a small portion of patients showed a significant decrease in LVEF (4/22). According to the results, after anthracycline chemotherapy, the patients with DLBCL had abnormal regional myocardial perfusion, which was characterized by an increased SRS. As a much earlier indicator compared with LVEF and QTc interval, SRS could be used to evaluate the cardiac toxicity associated with LV dysfunction not only caused by chemotherapy (decreased LVEF) at an early stage, but also caused by chemotherapy-induced arrhythmia at an early stage. Such a conclusion might be attributed to abnormal perfusion being the pathological basis for the impairment of LV myocardial function. The severity of perfusion abnormality is closely associated with the decline in LV function. The detected value of LVEF might still be within the normal range when slight myocardial perfusion abnormalities occur. Therefore, compared with LVEF, SRS could evaluate cardiotoxicity at an earlier stage. Cardiomyocyte damage could cause abnormalities in myocardial electrical conduction and myocardial perfusion at the same time, while the sequence and mechanisms underlying these two types of abnormalities remain unclear. In the present study, the SRS abnormalities occurred earlier than QTc interval prolongation, indicating that myocardial mitochondrial damage after chemotherapy might first manifest as abnormal regional myocardial perfusion, which could be one of the factors causing arrhythmia. Studies have reported that patients with abnormal LV myocardial perfusion have a significantly higher incidence of arrhythmia (53). Therefore, compared with the QTc interval, SRS could predict the cardiac toxicity-associated arrhythmia caused by chemotherapy at an earlier stage.

There are several limitations to the present study. First, the sample size of this study was relatively small, and only 36 DLBCL patients were included. In the future, expansion of the sample size could continue, and a larger sample and more convincing data should be obtained. Second, the follow-up time was not long enough. In this study, the cardiac function of patients with DLBCL was monitored and compared only at the initial diagnosis and after six courses of R-CHOP chemotherapy. There may be some patients with delayed cardiac function. Therefore, the myocardial function of these patients should be followed up for longer and the follow-up results should be improved by rechecking G-MPI, color Doppler echocardiography, ECG and serum indices associated with myocardial function every 6 months. Third, the present study did not compare cardiac function among subgroups of patients with DLBCL with different prognostic stratifications due to the small sample size. DLBCL is a heterogeneous disease. Patients of different subtypes have very different prognoses. In the future, the number of patients with DLBCL should be expanded and the research refined further. In this way, cardiac function could be compared among different subgroups of patients with DLBCL.

In addition, complete and accurate data of event-free curves could not be provided for all cardiac events in these patients. Some patients were not able to visit the hospital for accurate cardiovascular event screening, and a small number of other patients were lost to follow-up for communication reasons. Missing data due to these objective reasons may affect the accuracy of the results of event-free curve analysis. We are currently in the process of expanding the number of patients 
enrolled and hope to complete an effective follow-up of all patients soon and analyze the relevant data.

In conclusion, the present study found that anthracycline chemotherapy was closely associated with the cardiotoxicity that occurred in the patients with DLBCL, especially the abnormal electrical conduction of the myocardium. The G-MPI SRS measurement was a highly sensitive method for detecting early subclinical cardiac damage to myocardial electrical conduction in patients with DLBCL treated with anthracyclines, which may be helpful for hematologists to monitor and treat the cardiac dysfunction of patients with DLBCL in a much more timely and convenient manner.

To the best of our knowledge, the present study was the first to use resting G-MPI to evaluate abnormal changes in LV myocardial perfusion in patients with DLBCL before and after chemotherapy. The G-MPI SRS level was an early indicator for TRC surveillance in patients with DLBCL after anthracycline chemotherapy, thus contributing to early treatment and a subsequent decrease in mortality caused by cardiovascular complications.

\section{Acknowledgements}

The authors would like to thank Dr Changqing Lu and Dr Tongbin Chen (The Third Affiliated Hospital of Soochow University, Changzhou, China) for providing technological assistance.

\section{Funding}

This study was supported by the Youth Science Fund Project of the National Natural Science Foundation of China (grant nos. 81800100 and 81701737), the National Natural Science Foundation of China (grant no. 81871381), the Youth Science Fund Project of the National Natural Science Foundation of Jiangsu, China (grant no. BK20150253), the Fund of 333 Project of Jiangsu, China (grant no. BRA2015088) and the Social Development Foundation of Changzhou Science and Technology Bureau, Jiangsu, China (grant no. CE20175029).

\section{Availability of data and materials}

The datasets used and/or analyzed during the current study are available from the corresponding author on reasonable request.

\section{Authors' contributions}

YL and JW conceived and designed the study. MX, CQ, PX, LS, BH, FW, YY, YG, FL and WD performed all the clinical diagnoses and treatments. YL and PX analyzed the data. YL, JW and PX wrote the manuscript. XX contributed to statistical analysis and revised the content of manuscript. YW and WG collected the clinicopathological data, made the tables and figures, and supervised the research group. All authors have read and approved the final manuscript.

\section{Ethics approval and consent to participate}

All procedures were in accordance with the ethical standards of the responsible human experimentation committee (institutional and national) and the Declaration of Helsinki (1975), as amended in 2008. Patient samples were obtained with approval from the Ethical Committee at the First People's Hospital of Changzhou (Changzhou, China; approval number, CL022-01), and written informed consent was obtained from all patients according to the institutional guidelines.

\section{Patient consent for publication}

Not applicable.

\section{Competing interests}

The authors declare that they have no competing interests.

\section{References}

1. Li S, Young KH and Medeiros LJ: Diffuse large B-cell lymphoma. Pathology 50: 74-87, 2018.

2. Miyazaki K: Treatment of diffuse large B-cell lymphoma. J Clin Exp Hematop 56: 79-88, 2016.

3. Kubuschok B, Held G and Pfreundschuh M: Management of diffuse large B-cell lymphoma (DLBCL). Cancer Treat Res 165: 271-288, 2015.

4. Cardinale D, Colombo A, Bacchiani G, Tedeschi I, Meroni CA, Veglia F, Civelli M, Lamantia G, Colombo N, Curigliano G, et al: Early detection of anthracycline cardiotoxicity and improvement with heart failure therapy. Circulation 131: 1981-1988, 2015.

5. Linschoten M, Teske AJ, Baas AF, Vink A, Dooijes D, Baars HF and Asselbergs FW: Truncating Titin (TTN) variants in chemotherapy-induced cardiomyopathy. J Card Fail 23: 476-479, 2017.

6. Ewer MS and Ewer SM: Cardiotoxicity of anticancer treatments. J Nat Rev Cardiol 12: 547-558, 2015.

7. Laursen AH, Thune JJ, Hutchings M, Hasbak P, Kjaer A, Elming MB and Ripa RS: ${ }^{123}$ I-MIBG imaging for detection of anthracycline-induced cardiomyopathy. Clin Physiol Funct Imaging 38: 176-185, 2018.

8. Mcmurray JJ Adamopoulos S, Anker SD, Auricchio A, Böhm M, Dickstein K, Falk V, Filippatos G, Fonseca C, Gomez-Sanchez MA, et al: ESC Guidelines for the diagnosis and treatment of acute and chronic heart failure 2012: The Task Force for the diagnosis and treatment of acute and chronic heart failure 2012 of the European Society of Cardiology. Developed in collaboration with the Heart Failure Association (HFA) of the ESC. Eur J Heart Fail 14: 803-869, 2012.

9. Dutta T, Spevack DM and Aronow WS: The left ventricular ejection fraction: New insights into an old parameter. Hosp Pract (1995) 47: 221-230, 2019.

10. Ong G, Brezden-Masley C, Dhir V, Deva DP, Chan KKW, Chow CM, Thavendiranathan D, Haq R, Barfett JJ, Petrella TM, et al: Myocardial strain imaging by cardiac magnetic resonance for detection of subclinical myocardial dysfunction in breast cancer patients receiving trastuzumab and chemotherapy. Int J Cardiol 261: 228-233, 2018

11. Virani SA, Dent S, Brezden-Masley C, Clarke B, Davis MK, Jassal DS, Johnson C, Lemieux J, Paterson I, Sebag IA, et al: Canadian cardiovascular society guidelines for evaluation and management of cardiovascular complications of cancer therapy. Can J Cardiol 32: 831-841, 2016.

12. Zamorano JL, Lancellotti P,Muñoz DR, Aboyans V, Asteggiano R, Galderisi M, Habib G, Lenihan DJ, Lip GY, Lyon AR, et al: 2016 ESC Position Paper on cancer treatments and cardiovascular toxicity developed under the auspices of the ESC Committee for Practice Guidelines. Kardiol Pol 74: 1193-1233, 2016 (In Polish).

13. Seraphim A, Westwood M, Bhuva AN, Crake T, Moon JC, Menezes LJ, Lloyd G, Ghosh AK, Slater S, Oakervee H and Manisty $\mathrm{CH}$ : Advanced imaging modalities to monitor for cardiotoxicity. Curr Treat Options Oncol 20: 73, 2019.

14. Markman TM, Ruble K, Loeb D, Chen A, Zhang Y, Beasley GS, Thompson WR and Nazarian S: Electrophysiological effects of anthracyclines in adult survivors of pediatric malignancy. Pediatric Blood Cancer: 64, 2017 doi: 10.1002/pbc.26556.

15. Yu AF, Manrique C, Pun S, Liu JE, Mara E, Fleisher M, Patil S, Jones LW, Steingart RM, Hudis CA and Dang CT: Cardiac safety of paclitaxel plus trastuzumab and pertuzumab in patients with HER2-positive metastatic breast cancer. Oncologist 21: 418-424, 2016. 
16. Soufer A, Liu C, Henry ML and Baldassarre LA: Nuclear cardiology in the context of multimodality imaging to detect cardiac toxicity from cancer therapeutics: Established and emerging methods. J Nucl Cardiol 27: 1210-1224, 2020.

17. Mahabadi AA and Rischpler C: Cardiovascular imaging in cardio-oncology. J Thorac Dis 10 (Suppl 35): S4351-S4366, 2018

18. Gayed IW, Liu HH, Yusuf SW, Komaki R, Wei X, Wang X, Chang JY, Swafford J, Broemeling L and Liao Z: The prevalence of myocardial ischemia after concurrent chemoradiation therapy as detected by gated myocardial perfusion imaging in patients with esophageal cancer. J Nucl Med 47: 1756-1762, 2006.

19. Zellars R, Bravo PE, Tryggestad E, Hopfer K, Myers L, Tahari A, Asrari F, Ziessman H and Garrett-Mayer E: SPECT analysis of cardiac perfusion changes after whole-breast/chest wall radiation therapy with or without active breathing coordinator: Results of a randomized phase 3 trial. Int J Radiat Oncol Biol Phys 88: $778-785,2014$.

20. Germano G, Kavanagh PB, Slomka PJ, Van Kriekinge SD, Pollard G and Berman DS: Quantitation in gated perfusion SPECT imaging: The Cedars-Sinai approach. J Nucl Cardiol 14: 433-454, 2007.

21. King JF and Lam JT: A practical approach to diagnosis of B-cell lymphomas with diffuse large cell morphology. Arch Pathol Lab Med 144: 160-167, 2020.

22. Sabattini E, Bacci F, Sagramoso C and Pileri SA: WHO classification of tumours of haematopoietic and lymphoid tissues in 2008: An overview. Pathologica 102: 83-87, 2010.

23. Rosenberg SA: Validity of the Ann Arbor staging classification for the non-Hodgkin's lymphomas. J Cancer Treat Rep 61: $1023-1027,1977$.

24. Perez EA, Suman VJ, Davidson NE, Sledge GW, Kaufman PA, Hudis CA, Martino S, Gralow JR, Dakhil SR, Ingle JN, et al: Cardiac safety analysis of doxorubicin and cyclophosphamide followed by paclitaxel with or without trastuzumab in the North Central Cancer Treatment Group N9831 adjuvant breast cancer trial. J Clin Oncol 26: 1231-1238, 2008.

25. Goel S, Liu J, Guo H, Barry W, Bell R, Murray B, Lynch J, Bastick P, Chantrill L, Kiely BE, et al: Decline in left ventricular ejection fraction following anthracyclines predicts trastuzumab cardiotoxicity. JACC. Heart Fail 7: 795-804, 2019.

26. Ho E, Brown A, Barrett P, Morgan RB, King G, Kennedy MJ and Murphy RT: Subclinical anthracycline- and trastuzumab-induced cardiotoxicity in the long-term follow-up of asymptomatic breast cancer survivors: A speckle tracking echocardiographic study. Heart 96: 701-707, 2010

27. Yu W, Li SN, Chan GC, Ha SY, Wong SJ and Cheung YF: Transmural strain and rotation gradient in survivors of childhood cancers. Eur Heart J Cardiovasc Imaging 14: 175-182, 2013.

28. Armstrong IS, Arumugam P, James JM, Tonge CM and Lawson RS: Reduced-count myocardial perfusion SPECT with resolution recovery. Nucl Med Commun 33: 121-129, 2012.

29. Cerqueira MD, Weissman NJ, Dilsizian V, Jacobs AK, Kaul S, Laskey WK, Pennell DJ, Rumberger JA, Ryan T and Verani MS American Heart Association Writing Group on Myocardial Segmentation and Registration for Cardiac Imaging: Standardized myocardial segmentation and nomenclature for tomographic imaging of the heart. A statement for healthcare professionals from the cardiac imaging committee of the council on clinical cardiology of the american heart association. J Nucl Cardiol 9: 240-245, 2002.

30. McGowan JV, Chung R, Maulik A, Piotrowska I, Walker JM and Yellon DM: Anthracycline chemotherapy and cardiotoxicity. Cardiovasc Drugs Ther 31: 63-75, 2017.

31. Ferraro MP, Gimeno-Vazquez E, Subirana I, Gómez M, Díaz J, Sánchez-González B, García-Pallarols F, Martínez L, Ble M, Molina L, et al: Anthracycline-induced cardiotoxicity in diffuse large B-cell lymphoma: NT-proBNP and cardiovascular score for risk stratification. Eur J Haematol 102: 509-515, 2019.

32. Zelenetz AD, Gordon LI, Abramson JS, Advani RH, Bartlett NL, Caimi PF, Chang JE, Chavez JC, Christian B, Fayad LE, et al: NCCN guidelines insights: B-cell lymphomas, version 3.2019. J Natl Compr Canc Netw 17: 650-661, 2019.

33. Thieblemont C, Bernard S, Meignan M and Molina T: Optimizing initial therapy in DLBCL. Best Pract Res Clin Haematol 31: 199-208, 2018.

34. Bansal N, Amdani S, Lipshultz ER and Lipshultz SE: Chemotherapy-induced cardiotoxicity in children. Expert Opin Drug Metab Toxicol 13: 817-832, 2017.

35. Veronese P, Hachul DT, Scanavacca MI, Hajjar LA, Wu TC, Sacilotto L, Veronese C and Darrieux FCDC: Effects of anthracycline, cyclophosphamide and taxane chemotherapy on QTc measurements in patients with breast cancer. PLoS One 13: e0196763, 2018.
36. Porta-Sanchez A, Gilbert C, Spears D, Amir E, Chan J, Nanthakumar K and Thavendiranathan P: Incidence, diagnosis, and management of qt prolongation induced by cancer therapies: A systematic review. J Am Heart Assoc 6: e007724, 2017.

37. Heemskerk CPM, Pereboom M, van Stralen K, Berger FA, van den Bemt PMLA, Kuijper AFM, van der Hoeven RTM, Mantel-Teeuwisse AK and Becker ML: Risk factors for QTc interval prolongation. Eur J Clin Pharmacol 74: 183-191, 2018.

38. Puppe J, van Ooyen D, Neise J, Thangarajah F, Eichler C, Krämer S, Pfister R, Mallmann P, Wirtz M and Michels G: Evaluation of QTc Interval prolongation in breast cancer patients after treatment with epirubicin, cyclophosphamide, and docetaxel and the influence of interobserver variation. Breast Care (Basel) 12: 40-44, 2017.

39. Etchegoyen CV, Keller GA, Mrad S, Cheng S and Di Girolamo G: Drug-induced QT interval prolongation in the intensive care unit. Curr Clin Pharmacol 12: 210-222, 2017.

40. Renu K, Abilash VG, Tirupathi PB and Arunachalam S: Molecular mechanism of Doxorubicin-induced cardiomyopathy-An update. Eur J Pharmacol 818: 241-253, 2018.

41. Henninger C and Fritz G: Statins in Anthracycline-induced cardiotoxicity: Rac and Rho, and the heartbreakers. Cell Death Dis 8: e2564, 2017.

42. Hahn VS, Lenihan DJ and Ky B: Cancer Therapy-induced cardiotoxicity: Basic mechanisms and potential cardioprotective therapies. J Am Heart Assoc 3: e000665, 2014.

43. Rochette L, Guenancia C, Gudjoncik A, Hachet O, Zeller M, Cottin Y and Vergely C: Anthracyclines/trastuzumab: New aspects of cardiotoxicity and molecular mechanisms. Trends Pharmacol Sci 36: 326-348, 2015.

44. Mukai M, Komori K and Oka T: Mechanism and management of cancer Chemotherapy-induced atherosclerosis. J Atheroscler Thromb 25: 994-1002, 2018.

45. Yin J, Guo J, Zhang Q, Cui L, Zhang L, Zhang T, Zhao J, Li J, Middleton A, Carmichael PL and Peng S: Doxorubicin-induced mitophagy and mitochondrial damage is associated with dysregulation of the PINK1/parkin pathway. Toxicol In Vitro 51: $1-10,2018$.

46. Koleini N and Kardami E: Autophagy and mitophagy in the context of Doxorubicin-induced cardiotoxicity. Oncotarget 8: 46663-46680, 2017.

47. Ichikawa Y, Ghanefar M, Bayeva M, Wu R, Khechaduri A, Naga Prasad SV, Mutharasan RK, Naik TJ and Ardehali H: Cardiotoxicity of doxorubicin is mediated through mitochondrial iron accumulation. J Clin Invest 124: 617-630, 2014.

48. Beller GA and Watson DD: Physiological basis of myocardial perfusion imaging with the technetium $99 \mathrm{~m}$ agents. Semin Nucl Med 21: 173-181, 1991

49. Li J, Lu J and Zhou Y: Mitochondrial-targeted molecular imaging in cardiac disease. Biomed Res Int 2017: 5246853, 2017.

50. Bartholoma MD, Zhang S, Akurathi V, Pacak CA, Dunning P, Fahey FH, Cowan DB, Treves ST and Packard AB: (18) F-labeled rhodamines as potential myocardial perfusion agents: Comparison of pharmacokinetic properties of several rhodamines. Nucl Med Biol 42: 796-803, 2015.

51. Zhang L, Liu Z, Hu KY, Tian QB, Wei LG, Zhao Z, Shen HR and $\mathrm{Hu}$ J: Early myocardial damage assessment in dystrophinopathies using (99) Tc(m)-MIBI gated myocardial perfusion imaging. Ther Clin Risk Manag 11: 1819-1827, 2015.

52. Arruda-Olson AM, Roger VL, Jaffe AS, Hodge DO, Gibbons RJ and Miller TD: Troponin T levels and infarct size by SPECT myocardial perfusion imaging. JACC Cardiovasc Imaging 4: 523-533, 2011.

53. Sood N, Al Badarin F, Parker M, Pullatt R, Jacobson AF, Bateman TM and Heller GV: Resting perfusion MPI-SPECT combined with cardiac 123I-mIBG sympathetic innervation imaging improves prediction of arrhythmic events in Non-ischemic cardiomyopathy Patients: Sub-study from the ADMIRE-HF trial. J Nucl Cardiol 20: 813-820, 2013.

This work is licensed under a Creative Commons Attribution-NonCommercial-NoDerivatives 4.0 International (CC BY-NC-ND 4.0) License. 